\title{
The Effects of Chair Height and Foot Position on Chronic Stroke Patients' Sit-To-Walk Movement
}

\author{
Youngeun Choi, MSc, PT ${ }^{1)}$, Jin Gang Her, PhD, PT ${ }^{1}{ }^{*}$, Jooyeon Ko, PhD, $\mathrm{PT}^{2}$, \\ Do Heung Ko, PhD, ST ${ }^{1)}$, Jihae Woo, MS, PT ${ }^{3)}$, David O’Sullivan, $\mathrm{PhD}^{4}$, \\ HeEsoo KIM, MSc, OT ${ }^{1)}$ \\ 1) Department of Rehabilitation Therapy, Hallym University: Okcheon-dong, Chuncheon-si, Gangwon- \\ do 200-702, South Korea. TEL: +82 33-248-1421 \\ 2) Bundang CHA Medical Center \\ 3) Department of Physical Therapy, Hallym College \\ 4) Department of Sport Science, Chung-Ang University
}

\begin{abstract}
Purpose] The purpose of the present study was to examine differences in the sequence of movements of stroke patients performing sit-to-walk (STW) among various foot positions and chair. [Subjects] The subjects of the present study were 11 stroke patients and a control group of 11 subjects, with no significant differences in sex, age, height, weight and body mass index between the two groups were randomly selected. [Methods] STW tasks were performed under four different conditions: high seat with feet forward, high seat with feet backward, normal seat with feet forward and normal seat with feet backward) with different foot positions and chair heights. The STW tasks were divided into four phases and the sequences of STW movements during the phases were analyzed. The phases were as follows: rise preparation, transition, primary gait initiation, and secondary gait initiation. [Results] In the condition of the high seat with feet backward, decreases in the center of mass (COM) horizontal momentum were the smallest at the time point of ending of the rise preparation phase, and moving speeds and distances between the COM and the center of pressure (COP) significantly increased at swing leg toe-off which is at the end of the primary gait initiation phase.[Conclusion] Changes in the movement pattern of COM horizontal momentum were the most efficient when rising from chair of a height of $120 \%$ of the leg length, and COM horizontal momentum was maintained until swing leg toe-off in this condition. When practicing STW functional movements in the clinical setting, the foot positions and chair heights necessary to maintain the sequence of movements should be considered. Key words: Sit-to-walk, Stroke
\end{abstract}

(This article was submitted Nov. 1, 2012, and was accepted Nov. 28, 2012)

\section{INTRODUCTION}

Stroke patients have difficulties in performing forwardoriented movements, such as bending forward, sit-to-stand (STS), and sit-to-walk (STW) ${ }^{1-3)}$. Among these, STW is a movement that is frequently made in daily living, such as when standing from a chair to go to get an object. When walking begins, that is, at the moment of taking the first step, forward momentum is maintained because a completely standing position has not yet been adopted ${ }^{4}$.

To perform STW, horizontal momentum is generated through trunk flexion, and to stand up from a chair, vertical momentum generated by the lower limbs is necessary ${ }^{5)}$. When stroke patients perform STW, they experience difficulties in the STS movements that need to be made before the initiation of walking, because they cannot produce the joint extension momentum and force for standing from the sitting position due to muscle weakness and postural control impairment ${ }^{6-9)}$.

The extension moment for standing is affected by foot

*To whom correspondence should be addressed.

E-mail: jghur7@empal.com position and chair height ${ }^{10-12)}$. If the feet are positioned behind the knees, the movement of the center of gravity to the point where the ground reaction force is applied, will decrease and the distance between the center of gravity and therefore the resulting ground reaction force is reduced, which will reduce the activity of the ankle dorsiflexor ${ }^{10)}$. With regard to chair height, if the hip joints are located relatively higher than the knee joints, the net extension moment required of the lower limbs is decreased and this will reduce the peak vertical force of the ground reaction force ${ }^{11,12)}$. These foot positions and chair heights reduce the mechanical demands on extension moments of the lower limbs in STS, and transition from STS to walking, and this reduction may affect the coupling of horizontal momentum and vertical momentum.

In a comparison between healthy older adults and healthy young adults, it was demonstrated that the healthy young adults showed a more smoother transition from the standing to walking, whereas the older group showed postural instability and their forward momentum was reduced ${ }^{13-15)}$. STW of patients with Parkinson disease showed they have problems with the sequence of movements and have decreased velocity of movement between seat-off and gait initiation ${ }^{16)}$. 
Recently, with improvements in laboratory environments and access to additional force plates have allowed researchers to observe STW in more detail by dividing it into four phases: rise preparation, transition, primary gait initiation, and secondary gait initiation. Recent results indicated that the phase of transition is delayed for stroke patients ${ }^{17}$.

As far as we know, there has been no study that addresses the effect of STW on the natural transition of sequential movement from STS to walking among studies on STW conducted so far. While STS is a functional mobility trained at the early stage of rehabilitation as a preliminary phase for movements, STW involves making the actual movements of standing and taking the first step. Despite STW movements being important for mobility training in clinical rehabilitation, studies of stroke patients' STW are insufficient. Therefore, the purpose of the present study was to examine the effects of foot positions and chair heights on the sequence of movements when stroke patients are performing STW.

\section{SUBJECTS AND METHODS}

The subjects were 11 stroke patients (five males and six females) and 11 normal persons (five males and six females) with no significant differences in sex, age, height, weight, and BMI between the two groups fo subjects (Table 1). Before the subjects participated in the experiment, the purpose of the study was explained and consent forms were obtained from all the subjects. The criteria for selection of the stroke patients among the subjects were as follows: more than 6 months post-stroke, able to walk $10 \mathrm{~m}$ independently indoors without aid and or foot-ankle orthosis, able to rise from a chair without using their arms and hands, ability to understand verbal instructions, and have no neurological defects other than stroke.

Kinetic data were obtained from three force plates (Kistler 9260AA6, Kistler Instruments AG, CH-8408 Winterthur, Switzerland). The sampling frequency was $1000 \mathrm{~Hz}$. One force plate was placed on the chair to measure the force exerted on the region beneath the buttocks. Two force plates, embedded in a walkway $(5 \mathrm{~m} \times 1.2 \mathrm{~m})$, were used to measure the force under the each foot. Three-dimensional kinematic data were recorded using an 8-camera system (Qualisys $\mathrm{AB}$, Sweden) which sampled 54 markers at $100 \mathrm{~Hz}$. Before the experiment, the subjects changed their clothes into tight sport pants and tops. The markers measured using the cameras were identified using the Qualisys Track Manager program, and kinetic and kinematic parameters were calculated in Visual 3D. All marker data were low-pass filtered using a fourth order Butterworth filter with a cut-off frequency of $6 \mathrm{~Hz}$.

The experiment began with the subjects in a sitting position on a height-adjustable chair without any back or armrest. The two chair heights were set: $100 \%$ and $120 \%$ of the lower leg length measured as the distance between the ground and the lateral epicondyle of the femur ${ }^{18,19)}$. Two foot positions were taken: anterior with ankle dorsiflexion at $0^{\circ}$ and posterior with dorsiflexion of $10^{\circ}$. The subjects performed sit-to-walk movements at self-selected speeds under each of the four different experimental conditions. The four
Table 1. Subject characteristics $(\mathrm{N}=22)$

\begin{tabular}{lcc}
\hline Variables & Stroke $(\mathrm{n}=11)$ & Control $(\mathrm{n}=11)$ \\
\hline Sex $($ female/male) & $5 / 6$ & $5 / 6$ \\
Age $(\mathrm{y})$ & $52.09 \pm 8.92$ & $53.82 \pm 8.62$ \\
Weight $(\mathrm{kg})$ & $69.27 \pm 12.28$ & $68.91 \pm 11.51$ \\
Height $(\mathrm{m})$ & $1.66 \pm 0.06$ & $1.67 \pm 0.08$ \\
BMI $\left(\mathrm{kg} / \mathrm{m}^{2}\right)$ & $24.89 \pm 2.74$ & $24.37 \pm 2.61$ \\
Side $(\mathrm{right} / \mathrm{left})$ & $4 / 7$ & $\mathrm{NA}$ \\
10m walking speed $(\mathrm{m} / \mathrm{s})$ & $0.83 \pm 0.22$ & $1.50 \pm 0.16$ \\
Timed Up and Go $(\mathrm{s})$ & $14.22 \pm 2.88$ & $7.35 \pm 1.21$ \\
\hline
\end{tabular}

Values are number or mean \pm SD. Abbreviation: BMI; body mass index; NA: not applicable.

experimental conditions were as follows: (1) High seat foot forward (HSFF), Standing to walk from a sitting position on a chair of a height of $120 \%$ of the lower leg length with the feet placed anterior to the knees and with ankle dorsiflexion at $0^{\circ}$, (2) High seat with feet backward (HSFB), STW from a sitting position on a chair of a height of $120 \%$ of the lower leg length with the feet placed posterior to the knees and with ankle dorsiflexion at $10^{\circ}$; (3) Normal seat with feet forward (NSFF), STW from a sitting position on a chair of a height of $100 \%$ of the lower leg length with the feet placed anterior to the knees and with ankle dorsiflexion at $0^{\circ}$; and (4) Normal seat with feet backward (NSFB). STW from a sitting position on a chair of a height of $100 \%$ of the lower leg length with the feet placed posterior to the knees and with ankle dorsiflexion at $10^{\circ}$.

Each subject was instructed to sit keeping his/her trunk upright in a straight line with placing the greater trochanter close to the edge of the chair. The subject's feet were placed parallel to each other at pelvic width as measured between the two lateral malleoli. To maintain these four conditions constant while the subject's movements were measured, five times per condition, tapes were applied to the force plates to indicate foot and thigh positions. A telephone set was placed at the end of the walkway to emulate a situation similar to situations in daily living, with the instruction: "The phone is ringing; please go and answer the phone". Each subject was instructed not to move his/her feet or use either of his/her hands while he/she was standing from the sitting position. The four conditions were measured in a random order. The subject's movements were measured five times per condition and out of the five measurements, three without any hidden marker were selected and used for anal$y \operatorname{sis}^{20)}$. A rest time was given when the movements of each condition were completed and was also given whenever the subject requested one.

Based on previous studies of STW ${ }^{17,21)}$, movement events were identified. The events were defined as follows: event 1 was defined as movement initiation, at the point crossing the threshold, $7 \%$ of the peak amplitude of the forward momentum of the COM was reached; event 2 was defined as seat-off, at the first point when no force was measured on the force plate beneath the buttocks; event 3 was defined as the point at which the vertical force measured on the force plate beneath the first swing leg was at the maximum; event 

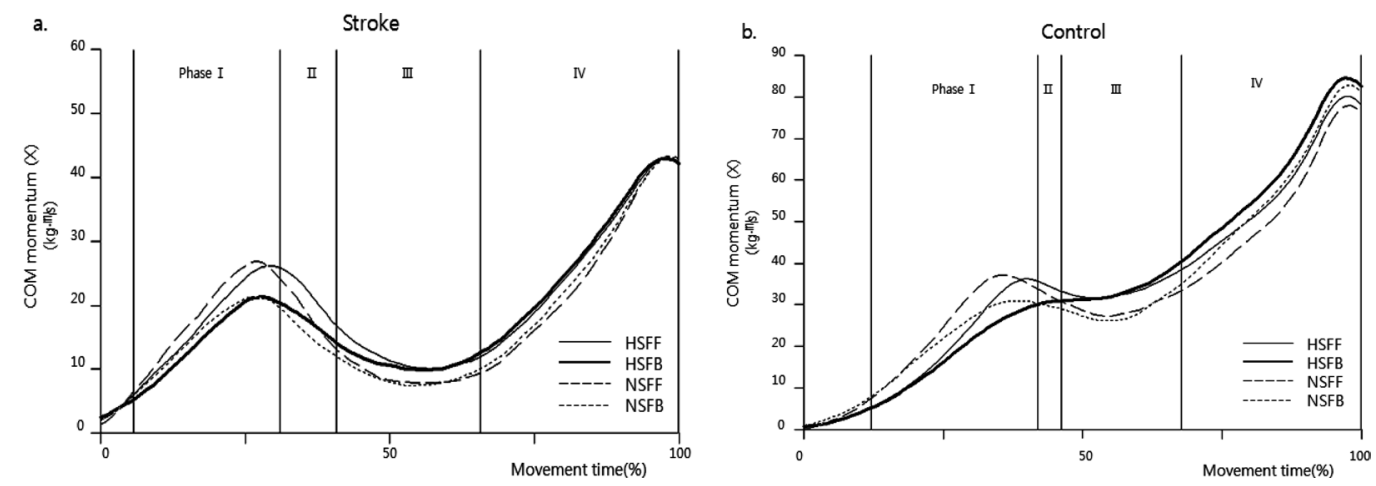

Fig. 1. The average trajectory of the body center of mass (COM) in the horizontal direction in the four sit-to-walk conditions (HSFF: high seat with feet forward, HSFB: high seat with feet backward, NSFF: normal seat with feet forward, NSFB: normal seat with feet backward) of stroke (a) and control subjects (b)

Table 2. Comparison of propulsion among the four STW conditions prior to seat-off

\begin{tabular}{lcll}
\hline Variable & Initial position & \multicolumn{1}{c}{ Stroke } & Control \\
\hline & HSFF & $0.25 \pm 0.13^{2}$ & $0.40 \pm 0.10^{2}$ \\
Propulsion (NS/kg) & HSFB & $0.18 \pm 0.12^{1,3}$ & $0.28 \pm 0.07^{1,3}$ \\
& NSFF & $0.29 \pm 0.12^{2,4}$ & $0.44 \pm 0.12^{2,4}$ \\
& NSFB & $0.21 \pm 0.14^{3}$ & $0.32 \pm 0.09^{3}$ \\
\hline
\end{tabular}

${ }^{1}$ is significant difference from $\mathrm{HSFF},{ }^{2}$ is significant difference from $\mathrm{HSFB},{ }^{3}$ is significant difference from NSFF, ${ }^{4}$ is significant difference from NSFB.

4 was defined as the point at which the vertical force disappeared as a result of the first swing leg toe-off; and event V was defined as the point at which the vertical force disappeared as a result of the stance leg toe-off of the leg that took the second step. Using the five events, the following four phases were defined: rise preparation phase; movement initiation $\sim$ seat-off; transition phase, seat-off $\sim$ swing leg loading peak; primary gait initiation phase; swing leg loading peak $\sim$ swing leg toe-off; and secondary gait initiation phase; and swing leg toe-off $\sim$ stance leg toe-off .

In the rise preparation phase, from the anterior-posterior force measured on the force plate beneath the buttocks, the propulsive impulse that indicates the forward component of the ground reaction force was calculated. In the transition phase, the vertical reaction force (VRF) measured under each foot at the point when the loading peak of the first swing leg occurred was measured to compare the index of asymmetry (IA) of the vertical reaction force between the affected and unaffected legs. The index of asymmetry was expressed as a percentage and its definition is as follows:

$\mathrm{IA}=\frac{\mathrm{VRF} \text { on the unaffected side }-\mathrm{VRF} \text { of perfect symmetry }}{\mathrm{VRF} \text { of perfect symmetry }} \times 100$

where, perfect symmetry is the value obtained by dividing the sum total of VRF by 2 .

In the primary gait initiation phase, the first step velocity at the point of swing leg toe-off was calculated using the COM horizontal velocity, and the COP was calculated using the center of pressure (COP) located on the force plate located beneath each foot ${ }^{22)}$.
Collected data were statistically analyzed using PASW 18.0. Repeated measures ANOVA were conducted to compare the effects of the four starting positions (NSFF, NSFB, HSFF, and HSFB). Bonferroni's method was used for the post-hoc tests, and the significance level was accepted for values of $p<0.05$.

\section{RESULTS}

The characteristics of COM horizontal momentum patterns of the stroke patients and the normal control group from the four different starting positions are shown in Fig. 1. In the control shown in (b), the COM horizontal momentum curve of HSFB shows that the momentum continuously increased until the peak and decreased a little when the movements reached a steady state. In the stroke group (a), the momentum rapidly decreased after the first peak in all of the four conditions and increased again immediately before the swing leg toe-off. Among the conditions, HSFB showed the slowest decreasing trend and NSFF showed the most rapid decrease.

The propulsion in the rise preparation phase was compared in order examine horizontal momentum. In the stroke patient group, the propulsion increased the most in HSFB followed by NSFB, HSFF, and NSFF in order of magnitude, and the control group showed increases in propulsion in the same sequence. HSFB showed significant decreases compared to NSFF and HSFF ( $<<0.05$ ) (Table 2).

In the transition phase, the IA at the swing leg loading peak was compared to identify the force distribution when lower limb vertical momentum was generated. Neither of 
Table 3. Index of asymmetry for the four STW conditions at swing leg loading peak

\begin{tabular}{cccc}
\hline Variable & Initial position & \multicolumn{1}{c}{ Stroke } & Control \\
\hline \multirow{3}{*}{ IA (\%) } & HSFF & $16.99 \pm 10.64$ & $-8.73 \pm 11.38$ \\
& HSFB & $14.55 \pm 9.44$ & $-13.24 \pm 10.11$ \\
& NSFF & $20.86 \pm 12.01$ & $6.78 \pm 6.45$ \\
& NSFB & $18.19 \pm 12.44$ & $6.10 \pm 3.24$ \\
\hline
\end{tabular}

IA: index of asymmetry

Table 4. Velocity and COM-COP moment arm of the STW conditions at swing leg toe-off

\begin{tabular}{lccc}
\hline Variable & Initial position & Stroke & Control \\
\hline \multirow{4}{*}{ Initial step velocity $(\mathrm{m} / \mathrm{s})$} & HSFF & $0.14 \pm 0.86^{2,3}$ & $0.49 \pm 0.08^{2}$ \\
& HSFB & $0.17 \pm 0.10^{1,3,4}$ & $0.66 \pm 0.05^{1,3,4}$ \\
& NSFF & $0.12 \pm 0.90^{1,2,4}$ & $0.44 \pm 0.08^{2,4}$ \\
& NSFB & $0.15 \pm 0.10^{2,3}$ & $0.52 \pm 0.07^{2,3}$ \\
& HSFF & $5.56 \pm 2.57^{2}$ & $9.58 \pm 1.32^{2,3,4}$ \\
COM-COP moment arm (cm) & HSFB & $6.16 \pm 2.73^{1,3}$ & $10.79 \pm 1.23^{1,3,4}$ \\
& NSFF & $4.92 \pm 2.80^{2,4}$ & $8.63 \pm 1.36^{1,2,4}$ \\
& NSFB & $5.78 \pm 2.73^{3}$ & $10.19 \pm 1.12^{1,2,3}$ \\
\hline
\end{tabular}

${ }^{1}$ is significant difference from HSFF; ${ }^{2}$ is significant difference from HSFB $;{ }^{3}$ is significant difference from NSFF; ${ }^{4}$ is significant difference from NSFB.

the two groups showed significant differences between the starting positions (Table 3).

In the primary gait initiation phase, the speed of the swing leg and the distance between the COM and the COP at the point of swing leg toe-off were compared to see whether forward momentum was maintained. For the stroke patients, the speed and the distance between the COM and the COP decreased the most in HSFB followed by NSFB, HSFF, and NSFF in order of magnitude and the control group also showed decreases in the speed and the distance in the same sequence. The initial step velocity in HSFB was significantly increased compared to HSFF, NSFB, and NSFF $(p<0.05)$. The distance between the COM and the COP in HSFB was significantly decreased compared to HSFF and NSFF $(p<0.05)$ (Table 4).

\section{DISCUSSION}

The present study analyzed the effects of foot position and chair height on the sequence of movements of stroke patients when they performed STW tasks. According to the results, horizontal momentum was maintained best in the STS part of STW, and from standing to gait initiation at a chair height of $120 \%$ of the length of the lower limb and with the feet posterior to the knees. For an efficient initial step after standing, sufficient horizontal momentum needs to be generated before seat-off, to create a mechanism like an inverted pendulum, and the stance leg that takes the second step needs to serve the function of the pendulum to minimize the energy consumed in the initial step to be $\operatorname{made}^{5)}$.

In the pattern of COM horizontal momentum in the stroke group, the momentum decreased after the first peak and began to increase immediately before the swing leg toe-off, and decreased a little immediately before stance leg toe-off when movements were stabilized. Among the curves of the conditions, the HSFB curve showed the smallest difference between the first maximum value and the minimum value compared to the other three curves and was the closest to fluid movement among the four conditions. In STS, the body center of mass moves forward and upward toward a new reduced basal surface and the forward momentum stops in the standing position, while in STW the horizontal velocity is maintained ${ }^{23}$. When a person experiences difficulties in performing STW, he/she performs STS first before beginning walking. As the velocity of walking increases and the abilities of balance and movements improve; that is, as functional recovery progresses, body movements during STW become more fluid ${ }^{24)}$. Placing the feet posterior to the knee reduces the distance of the movement of COM, to enabling more efficient movements during standing ${ }^{10)}$, and having the chair higher than the length of the lower limb reduces the movement of the COM and the knee extension moment. The position of HSFB, in which vertical and horizontal movements of the COM are easy, helped the subjects make the first step more efficiently ${ }^{25)}$.

With regard to propulsion in the rise preparation phase, the largest propulsion was shown in NSFF while the smallest propulsion was shown in HSFB. With regard to the momentum-impulse relationship, propulsive impulses are necessary for the generation of forward momentum ${ }^{26}$. We stimulate the reason why propulsion was the largest in NSFF is thought to be the fact that the large horizontal movement distance of the COM required a larger force to bring the COM to the base of support.

Although vertical reaction forces (VRFs) and the indexes of asymmetry in the transition phase did not show any significant differences among the starting positions, such as 
different starting feet positions and chair heights, the index of asymmetry was the lowest in HSFB. If asymmetry increases, larger forces may be required for lower limb exten$\operatorname{sion}^{27)}$. Therefore, increasing the chair height for patients with severe motor impairment may help to maintain the sequence of STW.

The velocity of movements and COM-COP momentum at the start of the first swing, which is the end point of the primary gait initiation phase, increased the most in HSFB. This means that the forward momentum was maintained and supported the movement pattern of the COM horizontal moment arm in HSFB. The COM-COP moment arm is related to the ability of the person to control dynamic instability, and is a valid tool for identifying balance disorders ${ }^{28,29)}$. The difference in COM-COP distances between HSFB and NSFF is over $1 \mathrm{~cm}$ and thus HSFB showed a significant increase compared to NSFF. This seems to be a result of the continuous maintenance of forward momentum even after the seat-off.

The results of the present study indicate that when performing STW, the sequence which is characteristic of efficient STW can be obtained by adjusting the chair height and feet position of the starting position. For stroke patients to perform STW movements efficiently in daily living, feet positions and chair heights should be considered, when practicing STW in a clinical setting as a rehabilitation program, the sequence should be maintained through functional mobility training. This study focused on the effect that chair height and foot position have on STW; however, in future studies, the speed of the STW movement should also be considered.

\section{REFERENCES}

1) Chern JS, Lo CY, Wu CY, et al.: Dynamic postural control during trunk bending and reaching in healthy adults and stroke patients. Am J Phys Med Rehabil, 2010, 89: 186-197. [Medline] [CrossRef]

2) Cameron DM, Bohannon RW, Garrett GE, et al.: Physical impairments related to kinetic energy during sit to stand and curb climbing following strok. Clin Biomech (Bristol, Avon), 2003, 18: 332-340. [Medline] [CrossRef]

3) Dion L, Malouin F, McFadyen B, et al.: Assessing mobility and locomoto coordination after stroke with the rise to walk task. Neurorehabil Neural Repair, 2003, 17: 83-92. [Medline] [CrossRef]

4) Magnan A, McFadyen BJ, St-Vincent G: Modification of the sit to stand task with the addition of gait initiation. Gait Posture, 1996, 4: 232-241. [CrossRef]

5) Brenière Y, Do MC: Control of gait initiation. J Mot Behav, 1991, 23: 235 240. [Medline] [CrossRef]

6) Lomaglio MJ, Eng JJ: Muscle strength and weight bearing symmetry relate to sit to stand performance in individuals with stroke. Gait Posture, 2005, 22: 126-131. [Medline] [CrossRef]

7) Chou SW, Wong AM, Leong CP, et al.: Postural control during sit-to stand and gait in stroke patients. Am J Phys Med Rehabil, 2003, 82: 42-47. [Medline] [CrossRef]

8) Hanke TA, Pai YC, Rogers MW: Reliability of measurements of body center of mass momentum during sit-to-stand in healthy adults. Phys Ther 1995, 75: 105-113. [Medline]
9) Cheng PT, Chen CL, Wang CM, et al.: Leg muscle activation patterns of sit to stand movement in stroke patients. Am J Phys Med Rehabil, 2004, 83: 10-16. [Medline] [CrossRef]

10) Kawagoe S, Tajima N, Chosa E: Biomechanical analysis of effects of foot placement with varying chair height on the motion of standing up. J Orthop Sci, 2000, 5: 124-133. [Medline] [CrossRef]

11) Shepherd RB, Koh HP: Some biomechanical consequence of varying foot placement in sit-to-stand in young women. Scand J Rehabil Med, 1996, 28: 79-88. [Medline]

12) Rocha AS, Knabben RJ, Michaelsen SM: Non paretic lower limb constraint with a step decreases the asymmetry of vertical forces during sit to stand at two heights in subjects with hemiparesis. Gait Posture, 2010, 32: 457-463. [Medline] [CrossRef]

13) Buckley T, Pitsikoulis C, Barthelemy E, et al.: Age impairs sit to walk motor performance. J Biomech, 2009, 42: 2318-2322. [Medline] [CrossRef]

14) Kouta M, Shinkoda K, Shimizu ME: Biomechanical analysis of the sit to walk series of motions frequently observed in daily living: effects of motion speed on elderly persons. J Phys Ther Sci, 2007, 19: 267-271. [CrossRef]

15) Kouta M, Shinkoda K: Differences in biomechanical characteristics of sit to walk motion between younger and elderly males dwelling in the community. J Phys Ther Sci, 2008, 20: 185-189. [CrossRef]

16) Buckley TA, Pitsikoulis C, Hass CJ: Dynamic postural stability during sit to walk transitions in parkinson disease patients. Mov Disord, 2008, 23: 1274-1280. [Medline] [CrossRef]

17) Frykberg GE, Aberg AC, Halvorsen K, et al.: Temporal coordination of the sit to walk task in subjects with stroke and in controls. Arch Phys Med Rehabil, 2009, 90: 1009-1017. [Medline] [CrossRef]

18) Weiner DK, Long R, Hughes MA, et al.: When older adults face the chair rise challenge. A study of chair height availability and height modified chair-rise performance in the elderly. J Am Geriatr Soc, 1993, 41: 6-10. [Medline]

19) Rodosky MW, Andriacchi TP, Andersson GB: The influence of chair height on lower limb mechanics during rising. J Orthop Res, 1989, 7: $266-$ 271. [Medline] [CrossRef]

20) Mullineaux DR, Bartlett RM, Bennett $S$ : Research design and statistics in biomechanics and motor control. J Sports Sci, 2001, 19: 739-760. [Medline] [CrossRef]

21) Frykberg GE, Thierfelder T, Aberg AC, et al.: Impact of stroke on anteriorposterior force generation prior to seat off during sit to walk. Gait Posture, 2012, 35: 56-60. [Medline] [CrossRef]

22) Winter DA, Patla AE, Ishac M, et al.: Motor mechanisms of balance during quiet standing. J Electromyogr Kinesiol, 2003, 13: 49-56. [Medline] [CrossRef]

23) Vander Linden DW, Brunt D, McCulloch MU: Variant and invariant characteristics of the sit to stand task in healthy elderly adults. Arch Phys Med Rehabil, 1994, 75: 653-660. [Medline] [CrossRef]

24) Malouin F, McFadyen B, Dion L, et al.: A fluidity scale for evaluating the motor strategy of the rise to walk task after stroke. Clin Rehabil, 2003, 17: 674-684. [Medline] [CrossRef]

25) Mathiyakom W, McNitt-Gray JL, Requejo P, et al.: Modifying center of mass trajectory during sit to stand tasks redistributes the mechanical demand across the lower extremity joints. Clin Biomech (Bristol, Avon), 2005, 20: 105-111. [Medline] [CrossRef]

26) Pai YC, Rogers MW: Control of body mass transfer as a function of speed of ascent in sit to stand. Med Sci Sports Exerc, 1990, 22: 378-384. [Medline]

27) Roy G, Nadeau S, Denis G, et al.: Side difference in the hip and knee joint moments during sit to stand and stand to sit tasks in individuals with hemiparesis. Clin Biomech (Bristol, Avon), 2007, 22: 795-804. [Medline] [CrossRef]

28) Hass CJ, Waddell DE, Fleming RP, et al.: Gait initiation and dynamic balance control in parkinson's disease. Arch Phys Med Rehabil, 2005, 86: 2172-2176. [Medline] [CrossRef]

29) Hass CJ, Waddell DE, Wolf SL, et al.: Gait initiation in older adults with postural instability. Clin Biomech (Bristol, Avon), 2008, 23: 743-753. [Medline] [CrossRef] 